\title{
Gravitational waves from first order phase transitions
}

Chiara Caprini* ${ }^{* \dagger}$

Institut de Physique Théorique

CNRS, URA 2306 \& IPhT, CEA Saclay

91191 Gif-sur-Yvette, France

E-mail: chiara.capriniecea.fr

\begin{abstract}
Gravitational waves can constitute a unique probe of the very early universe. We discuss here the gravitational wave signal from a primordial first order phase transition occurring at high energy scales. The gravitational waves are produced by the collision of broken phase bubbles and by the magnetohydrodynamic turbulence in the primordial plasma stirred by the bubble collision. We show how the shape and amplitude of the gravitational wave power spectrum can be predicted by general arguments based on the source properties, such as its time evolution and space structure. The parameters describing the characteristics of the phase transition (for instance, its duration and strength), which enter in the determination of the gravitational wave spectrum, can be evaluated in the context of specific models of the first order phase transition. We present here two examples of first order phase transitions which lead to a gravitational wave signal falling in the frequency range of detection of the future space-based interferometer eLISA.
\end{abstract}

The European Physical Society Conference on High Energy Physics -EPS-HEP2013

18-24 July 2013

Stockholm, Sweden

\footnotetext{
*Speaker.

${ }^{\dagger}$ I wish to thank Pierre Binetruy, the organizer of the Cosmology parallel session, for the invitation.
} 


\section{Introduction}

In the course of its adiabatic expansion, the universe might have undergone several phase transitions (PT) driven by the temperature decrease. The nature of the primordial PT depends on the particle theory model, but if they are first order they proceed through the nucleation of broken phase bubbles, which is a very violent and inhomogeneous process capable of sourcing gravitational waves (GW). GW produced in the early universe form a fossil radiation: expansion prevents them from coming in thermal equilibrium with the other components of the universe because of the weakness of the gravitational interaction. Important information on the nature of the GW source is thus imprinted in relic GW and can be decoded. The detection of a fossil background of GW from a primordial PT would constitute a probe of the high energy processes giving rise to the PT and ultimately of the nature of the theoretical model describing the universe at high energy scales, possibly higher than those that can be reached in terrestrial laboratories. For example, the electro-weak phase transition (EWPT) in the standard model is a crossover, and it is not expected to lead to any appreciable cosmological signal; however, deviations from the standard model in the Higgs sector or the introduction of supersymmetry can lead to a first order EWPT. If the EWPT is sufficiently strong, it could lead to a GW signal detectable by the space-based interferometer eLISA [1]. Similarly, the QCDPT is also predicted to be a crossover by lattice simulations but it can become first order if the neutrino chemical potential is sufficiently large [2]. GW detection would help to probe the nature of these PT, and provide interesting information on the underlying particle theory.

\section{Overview of the GW signal: characteristic frequency and spectral shape}

In a cosmological context, GW may be represented by a tensor perturbation $h_{i j}(i, j=1,2,3)$ of the Friedmann Robertson-Walker metric ${ }^{1} d s^{2}=-d t^{2}+a^{2}(t)\left(\delta_{i j}+h_{i j}\right) d x^{i} d x^{j}$ which is transverse and traceless $\partial_{i} h_{i j}=h_{i i}=0$. The transverse-traceless condition leaves only two independent degrees of freedom, which are the ones that propagate and carry energy out of a source. In Fourier space, their linearized equation of motion given by Einstein equations is $\ddot{h}_{i j}(\mathbf{k}, t)+3 H \dot{h}_{i j}(\mathbf{k}, t)+$ $k^{2} h_{i j}(\mathbf{k}, t)=16 \pi G \Pi_{i j}^{(T T)}(\mathbf{k}, t)$, where $G$ is the Newton constant, $H$ is the Hubble rate, a dot denotes derivative with respect to $t, k$ is the physical wavenumber and $\Pi_{i j}^{(T T)}$ is the transverse-traceless part of the anisotropic stress $\Pi_{i j}$. The latter is given by $a^{2} \Pi_{i j}=T_{i j}-p a^{2}\left(\delta_{i j}+h_{i j}\right)$, where $T_{i j}$ denotes the spatial components of the energy-momentum tensor and $p$ is the background pressure. For a source operating at sub-Hubble scales $(k \gg H)$, the GW equation of motion may be approximated as a standard wave-equation $\ddot{h}_{i j}+k^{2} h_{i j}=16 \pi G T_{i j}^{(T T)} / a^{2}$. Once produced in the early universe, GW propagate freely until today, being simply redshifted by the expansion of the universe. Their energy density today can be written as $\rho_{g w}=\left\langle\dot{h}_{i j} \dot{h}_{i j}\right\rangle /(32 \pi G)=\int \frac{d f}{f} \frac{d \rho_{g w}}{d \log f}$, where $f=(k / 2 \pi)\left(a / a_{0}\right)$ is the present-day GW frequency ( $a_{0}$ denoting the scale factor today) and \langle\rangle denotes ensemble average. The superposition of GW produced by a large number of unresolved sources in the early universe form a stochastic background that is assumed to be statistically isotropic, stationary and nearly Gaussian. Its main properties are then described by its

\footnotetext{
${ }^{1}$ Where we assume flat spatial sections, $a(t)$ is the scale factor, $t$ denotes the physical time and repeated latin indices are summed.
} 
power spectrum. One defines the spectrum of energy density per logarithmic frequency interval divided by the critical density $\rho_{c}$ today, $h^{2} \Omega_{g w}(f)=\left(\frac{h^{2}}{\rho_{c}} \frac{d \rho_{g w}}{d \log f}\right)_{0}$, where the subscript 0 refers to the present epoch and $h$ parametrizes the small uncertainty in the value of the Hubble constant today, $H_{0}=100 h \mathrm{~km} / \mathrm{s} / \mathrm{Mpc}$.

A source that operates at sub-Hubble scales at some time $t_{*}$ after inflation emits GW with a characteristic wave-number $k_{*}$ that is larger than the Hubble rate $H_{*}$ at that time: $k_{*}=H_{*} / \varepsilon_{*}$ with $\varepsilon_{*} \leq 1$. The characteristic GW frequency today is then given by $f_{c}=\left(k_{*} / 2 \pi\right)\left(a_{*} / a_{0}\right)$. For GW produced in the radiation era when the plasma temperature is $T_{*}$, and assuming a standard adiabatic thermal history for the evolution of the universe after GW production, the characteristic frequency today can be written as $f_{c} \simeq 1.6 \times 10^{-4} \mathrm{~Hz} \varepsilon_{*}^{-1}\left(T_{*} / 1 \mathrm{TeV}\right)\left(g_{*} / 100\right)^{1 / 6}$, where $g_{*}$ is the number of relativistic degrees of freedom at temperature $T_{*}$. The parameter $\varepsilon_{*} \leq 1$ depends on the dynamics of the specific GW source under consideration. For a first-order phase transition, one may have for instance $\varepsilon_{*} \sim 10^{-3}-1$. In that case, GW produced around the EW scale are potentially interesting for detection with eLISA, which operates in the frequency range $10^{-5} \mathrm{~Hz}<f<1 \mathrm{~Hz}$ [1].

Towards the end of the PT, the true vacuum bubbles collide and convert the entire universe to the broken phase. The collisions break the spherical symmetry of the bubble walls, generating a non-zero anisotropic stress which acts as a source of GW. Moreover, bubble collision causes an injection of energy in the primordial plasma, which has a very high Reynolds number (of the order of $10^{13}$ at $100 \mathrm{GeV}$ and at the typical scale of the bubbles [3]): the energy injection leads to the formation of magnetohydrodynamic (MHD) turbulence, which sources GW through the anisotropic stresses of the chaotic fluid motions and of the magnetic field present in the plasma. Both GW generation processes are related to the collision of bubbles, which involves two quantities: the duration of the PT $\beta^{-1}$, and the typical size of the bubbles at the moment of collision, $R_{*} \simeq v_{b} \beta^{-1}$, where $v_{b}$ is the bubble wall velocity. The characteristic frequency of the GW generated by the two processes can correspond either to the duration of the PT or to the bubble size: $k_{*} \simeq \beta$ or $R_{*}^{-1}$, depending on the details of the time evolution of the source. These two parameters can therefore both determine the value of $\varepsilon_{*}$. If the growth of the bubble proceeds at a highly relativistic speed, the two time/length-scales are equal. Assuming for the moment $k_{*} \simeq \beta$, one obtains the following order of magnitude estimate of the characteristic frequency today:

$$
f \simeq 10^{-2} \frac{\beta}{H_{*}} \frac{T_{*}}{100 \mathrm{GeV}}\left(\frac{g_{*}}{100}\right)^{\frac{1}{6}} \mathrm{mHz} .
$$

The parameter $\beta / H_{*}$ is the ratio of the Hubble time to the duration of the PT. Since the entire universe must be converted to the broken phase, the PT must complete faster than a Hubble time, so in general we expect $\beta / H_{*}>1$. From Eq. (2.1) it appears that the characteristic frequency of GW emitted at the EWPT at $100 \mathrm{GeV}$ falls in the frequency range of eLISA for values $1 \lesssim \beta / H_{*} \lesssim 10^{4}$. As another example, we see from the above formula that GW production at the QCDPT at $T_{*} \simeq 100$ $\mathrm{MeV}$ can fall into the frequency range of detection with pulsar timing array, $f \geq 10^{-8} \mathrm{~Hz}$ (see e.g. [4] and references therein). The precise value of $\beta / H_{*}$ has to be determined in the context of a given model for the first order PT (c.f. section 3).

A simple estimate of the GW amplitude, which shows how the result scales with the duration and the energy density of the GW source, can be given through the following heuristic argument. We rewrite the GW equation of motion for $\beta / H_{*}>1$ simply as $\beta^{2} h \sim 16 \pi G T$, where $h$ denotes the 
amplitude of the tensor perturbation, $T$ the energy momentum tensor of the source, and we inserted $1 / \beta$ as the characteristic time on which the perturbation is evolving (we have dropped indices for simplicity). This suggests that $\dot{h} \sim 16 \pi G T / \beta$, and the GW energy density at the time of production can then be estimated as $\rho_{g w} \sim \dot{h}^{2} /(32 \pi G) \sim 8 \pi G T^{2} / \beta^{2}$. Dividing by the total energy density $\rho_{\text {tot }}=3 H_{*}^{2} /(8 \pi G)$ at the time of GW production, we can write $\rho_{g w} / \rho_{t o t} \sim\left(H_{*} / \beta\right)^{2}\left(\rho_{s} / \rho_{t o t}\right)_{*}^{2}$ where $\rho_{s} \sim T$ denotes the part of the energy density available in the source for the GW generation. Accounting for the fact that the PT takes place in the radiation-dominated universe and that the GW energy density is diluted like radiation, one then obtains, for the peak amplitude of the GW spectrum today

$$
\Omega_{g w} \sim \Omega_{R}\left(\frac{H_{*}}{\beta}\right)^{2}\left(\frac{\rho_{s}}{\rho_{t o t}}\right)_{*}^{2},
$$

where $\Omega_{R}=\rho_{R} / \rho_{c}$ denotes the radiation abundance today and $\left(\rho_{s} / \rho_{t o t}\right)_{*}$ the fraction of energy density that contributes to GW generation at the time of production. The above equation shows that the GW energy density scales like the square of the ratio of the GW source duration and the Hubble time, and the square of the energy density in the source. As a rule of thumb, given $h^{2} \Omega_{R} \simeq$ $4.15 \times 10^{-5}$, a GW signal above the lowest sensitivity of a typical detector, say $h^{2} \Omega_{g w} \gtrsim 10^{-10}$, can be generated if $\left(H_{*} / \beta\right)\left(\rho_{s} / \rho_{t o t}\right)_{*} \gtrsim 3 \times 10^{-3}$. Therefore, detectable signals arise from very energetic processes, which involve a sizable fraction of the total energy density in the universe, and at the same time slow processes, which minimize the value of $\beta / H_{*}$.

The slope of the GW spectrum at wave-numbers $k$ smaller than the Hubble radius $H_{*}$ at the time of production can also be determined on general grounds, valid for any transient stochastic source after inflation. This is a consequence of the fact that the causal process generating the $\mathrm{GW}$ signal cannot operate on time/length-scales larger than $1 / H_{*}$. Therefore, the anisotropic stresses $\Pi_{i j}^{(T T)}(\mathbf{k}, t)$ sourcing the metric perturbations are not correlated for $k<H_{*}$, and the anisotropic stress power spectrum is expected to be flat (white noise) up to the wavenumber $k_{*}$. If the anisotropic stress power spectrum is flat, so is expected to be the GW power spectrum $\left|\dot{h}_{i j}(\mathbf{k}, t)\right|^{2}$, because the causal horizon sets also the maximal time-scale of the correlation, and the wave equation cannot lead to any extra correlation for $k<H_{*}$. This in turn implies that the spectrum of GW energy density per logarithmic frequency interval must grow as $k^{3}$, because $d \rho_{g w} / d \log k \propto k^{3}\left|\dot{h}_{i j}(\mathbf{k}, t)\right|^{2}$. Thus the infra-red tail of the present-day GW spectrum behaves as $h^{2} \Omega_{g w} \propto f^{3}$ for scales that were super-Hubble at the time of production.

At sub-Hubble scales $k>H_{*}$, the spectrum continues to grow up to the characteristic wavenumber $k_{*}$. The slope depends on how long the GW source is active [3]. The anisotropic stresses due to bubble wall collisions disappear once the PT is completed: therefore, they act as a source of GW for less than a Hubble time. In this case, no correlations are generated beyond the characteristic wavenumber, and for $H_{*}<k<k_{*}$ the GW spectrum behaves as $k^{3}$. On the other hand, MHD turbulence takes several Hubble times before being finally dissipated (because of the very low viscosity of the primordial plasma [3]). Therefore, the anisotropic stresses due to the fluid motion and the magnetic field source GW for several Hubble times: this causes the slope of the GW signal to turn from $k^{3}$ to $k^{2}$ in the scale range $H_{*}<k<k_{*}$.

As previously mentioned, the characteristic scale $k_{*}$ at which the GW spectrum peaks corresponds either to the duration of the PT or to the bubble size $\left(k_{*} \simeq \beta\right.$ or $\left.R_{*}^{-1}\right)$, depending on the time 
correlation structure of the GW source. Bubble collision events are uncorrelated in time between each other, but each collision event has a deterministic time evolution. Under these conditions, it is possible to demonstrate [3] that the GW spectrum is given simply by the square of the time Fourier transform of the source. Consequently, it peaks at a wavenumber corresponding to the characteristic time-scale of the source, i.e. the total duration of the bubble collision process: $k_{*} \simeq \beta$. On the other hand, the time evolution of MHD turbulence cannot be established in a deterministic way: it is only known that turbulent motions de-correlate with a timescale corresponding to the eddy turnover time, which depends on the eddy size. This particular evolution structure does not lead directly to the time Fourier transform of the source of GW, and the peak of the GW spectrum changes to the characteristic length-scale of the source [3]: the size of the largest eddies, corresponding to the size of the bubbles at the end of the PT: $k_{*} \simeq R_{*}^{-1}$.

Beyond $k_{*}$, the GW power spectrum decays with a slope that depends on the details of the source power spectrum. For bubble collisions, it turns out that it decays as $1 / k$ [5]; for MHD turbulence instead, if it is of the Kolmogorov type, it leads to a GW spectrum decaying as $k^{-5 / 3}$ [3]. An example of the complete GW spectrum from a first order PT given by the sum of the bubble collision and MHD turbulence contributions is shown in the left plot of Fig. 1, were it is possible to appreciate the low and high frequency behaviors and the double peak structure.

\section{Parameters entering the GW spectrum}

In order to predict the amplitude and peak frequency of the GW signal from a specific first order PT one has to determine the value of the few parameters entering the GW spectrum. As we have seen, these are the PT temperature $T_{*}$, the inverse duration of the PT $\beta / H_{*}$, the bubble wall velocity $v_{b}$, and the fraction of energy that contributes to the GW generation $\left(\rho_{s} / \rho_{t o t}\right)_{*}$. These parameters are not all independent, and can only be determined within a given model of the PT. In general, one needs to find the bounce solution of the three-dimensional Euclidean action $S_{3}(T)$, which quantifies the probability of tunneling [6]. From this, one can then calculate the fraction of space that is covered by bubbles (neglecting overlap): $T_{*}$ can be defined as the temperature at which this fraction is equal to one. Moreover, knowing the three-action as a function of temperature, one can calculate $\beta / H_{*}=T d\left(S_{3} / T\right) / d T$, which is in general a function of temperature and has to be evaluated towards the end of the PT to represent, as a matter of fact, the 'duration' of the PT ${ }^{2}$.

The bubble wall velocity $v_{b}$ and the fraction of energy that contributes to the GW generation $\left(\rho_{s} / \rho_{t o t}\right)_{*}$ cannot in general be evaluated solely from the three-action. Since these two parameters are connected to the dynamics of the bubble expansion in the primordial fluid, a knowledge of the total particle content and interactions of the theory is in principle necessary to determine them. The bubble wall velocity $v_{b}$ results from the balance among the driving force that makes the bubble expand (given by the pressure difference between the interior and the exterior of the bubble, which is connected to the latent heat) and the friction force due to the interaction of the bubble wall with the surrounding plasma, which slows down the bubble expansion. This latter can either be determined in a given particle theory model, for which all interactions are known, or it can be parameterized in terms of a new parameter, the friction $\eta$, providing then a phenomenological

\footnotetext{
${ }^{2}$ Alternatively, it is possible to relate $\beta$ to the typical bubble size at the end of the PT through $v_{b},\langle R\rangle \simeq 3 v_{b} / \beta(T)$, where $\langle R\rangle$ can be estimated from the maximum of the bubble volume distribution [6].
} 

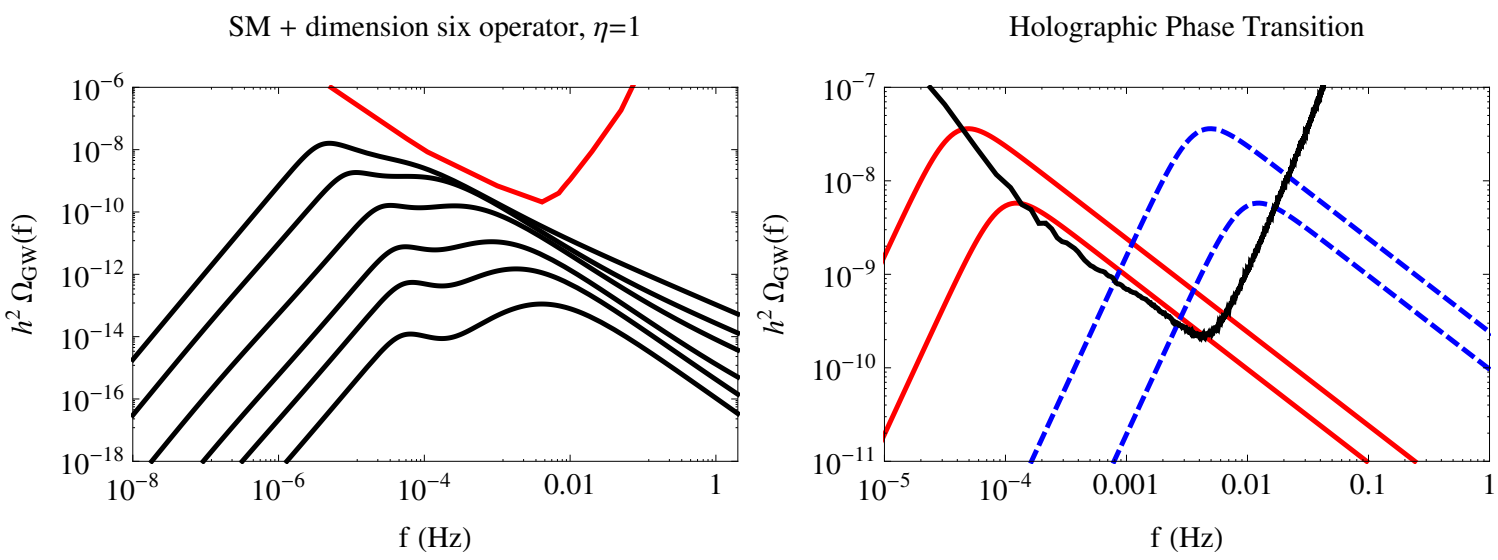

Figure 1: GW spectra in two models of first order PT, compared with the sensitivity curve of the interferometer eLISA. Left plot: the SM extended by a dimension 6 operator, with friction $\eta=1$. From bottom to top, the strength of the PT increases. Right plot: PT connected to the radion stabilization in the Randal Sundrum model (which is always in the runaway regime). The solid lines are the GW spectra for $T_{*}=100$ $\mathrm{GeV}$, and from top to bottom $\beta / H_{*}=6, \beta / H_{*}=15$; the dashed lines are for $T_{*}=10^{4} \mathrm{GeV}$, and the same values of $\beta / H_{*}$.

picture valid for several PT models [7]. Once $v_{b}$ is known, it gives the boundary condition for the hydrodynamical description of the bubble growth. The bubble can reach a steady state with constant velocity, with supersonic (detonation) or subsonic (deflagration) propagation speed; or, if the friction is subdominant with respect to the driving force, it can accelerate constantly up to the speed of light (runway solution) [7]. $\rho_{s}$ is in general given by the the sum of the gradient kinetic energy in the Higgs-like field driving the phase transition, the bulk kinetic energy of the fluid set into motion by the bubble wall, and the kinetic and magnetic energies due to the MHD turbulence. Either of these components can dominate $\rho_{s}$, depending on the characteristics of the PT and on the bubble propagation: if the friction is high, the bulk motions and the MHD turbulence dominate (e.g. in deflagrations); if on the contrary the friction is low, the transfer of energy from the bubble wall to the surrounding fluid is poor and most of the energy remains in the form of kinetic gradient energy of the Higgs-like field (e.g. in runaways).

To determine the parameter $\left(\rho_{s} / \rho_{\text {tot }}\right)_{*}$ entering the amplitude of the GW spectrum one further has to know the total energy density in the universe at the moment of the PT: $\rho_{\text {tot }}=\rho_{\text {rad }}^{*}+\rho_{\text {vac }}$, the sum of the radiation and the vacuum energy densities, of which the latter is known from the action in a given PT model. It is important to point out that the strength of the PT, customarily parameterized by the ratio $\alpha=\rho_{\mathrm{vac}} / \rho_{\text {rad }}^{*}$, is in general connected with its duration, parameterized by $\beta / H_{*}$ : strong PT last longer, leading to small $\beta / H_{*}$. This increases the amplitude of the GW signal (c.f. Eq. 2.2), but shifts the peak frequency to low values (c.f. Eq. 2.1).

\section{Discussion and conclusions}

In Fig. 1 we show two examples of the GW signal from first order PT [8]. The first example is the EWPT in the standard model augmented with dimension 6 operators [6], and with the phenomenological parameter representing the friction set to $\eta=1$. The GW signal increases with the 
strength of the PT, and correspondingly the peak frequency shifts to lower frequencies. The low frequency tail of the spectrum for $k<k_{*}$ raises as $k^{3}$. If the strength of the PT is low, the bulk kinetic energy dominates the GW production with respect to the kinetic gradient energy of the Higgs field: therefore, the peak of the GW spectrum is at $k_{*} \simeq R_{*}^{-1}$, set by the contribution from MHD turbulence. As the strength of the PT increases, the contribution from bubble collisions (which is determined by the gradient kinetic energy of the Higgs field) gains importance, and the peak of the GW spectrum becomes the inverse time-scale of the source, $k_{*} \simeq \beta$. Moreover, as the PT gets stronger, the temperature of the PT $T_{*}$ diminishes and the duration of the PT increases $\left(\beta / H_{*}\right.$ gets smaller): therefore, the peak of the GW spectrum is shifted to lower frequencies, progressively exiting the sensitivity range of the eLISA interferometer.

The second example is the GW signal in the holographic PT corresponding to the stabilization of the radion in the Randall Sundrum model [9]. This PT is characterized by a very high supercooling: the bubbles expand in the runaway regime (at the speed of light) and the fraction of energy that contributes to the GW generation is always given by the kinetic gradient energy of the scalar field. No MHD turbulence is present, and the spectrum always peaks at $k_{*} \simeq \beta$.

To summarize, we have little information about the physics and the processes operating in the very early universe, but it is in principle possible to generate a stochastic background of GW detectable by the next generation interferometers, which will reach an ultimate sensitivity around $10^{-10}$ in the GW energy density. In particular, the space-based interferometer eLISA will be sensitive to very strong PT occurring around $10 \mathrm{TeV}$ and which last long [8], such as for example PT connected to the presence of extra-dimensions and warped geometries. A well defined prediction of the spectral shape is crucial for establishing detection prospects, and to distinguish the signal from the noise. Some of the characteristics of the GW spectrum can be predicted on general grounds and from analytical estimates, such as the $k^{3}$ raise of the the large scale part of the spectrum, and the position of the spectrum peak in connection with the PT properties. In general, GW are a powerful mean to learn about the early universe and high energy physics: the detection is difficult, but it would lead to a great payoff.

\section{References}

[1] P. A. Seoane et al. [eLISA Collaboration], arXiv:1305.5720 [astro-ph.CO].

[2] D. J. Schwarz and M. Stuke, JCAP 0911 (2009) 025 [Erratum-ibid. 1010 (2010) E01] [arXiv:0906.3434 [hep-ph]].

[3] C. Caprini, R. Durrer and G. Servant, JCAP 0912 (2009) 024 [arXiv:0909.0622 [astro-ph.CO]].

[4] C. Caprini, R. Durrer and X. Siemens, Phys. Rev. D 82 (2010) 063511 [arXiv:1007.1218 [astro-ph.CO]].

[5] S. J. Huber and T. Konstandin, JCAP 0809 (2008) 022 [arXiv:0806.1828 [hep-ph]].

[6] S. J. Huber and T. Konstandin, JCAP 0805 (2008) 017 [arXiv:0709.2091 [hep-ph]].

[7] J. R. Espinosa et al, JCAP 1006 (2010) 028 [arXiv:1004.4187 [hep-ph]].

[8] P. Binetruy, A. Bohe, C. Caprini and J. -F. Dufaux, JCAP 1206 (2012) 027 [arXiv:1201.0983 [gr-qc]].

[9] L. Randall and G. Servant, JHEP 0705 (2007) 054 [hep-ph/0607158]; T. Konstandin and G. Servant, JCAP 1112 (2011) 009 [arXiv:1104.4791 [hep-ph]]. 